\title{
Überdiagnostik bei Gynäkomastie des Mannes
}

\author{
Bei der männlichen Gynäkomastie erfolgt viel zu häufig eine diagnostische Plethora, die an- \\ gesichts der Seltenheit relevanter pathologische Befunde in keinem Verhältnis zum möglichen \\ Ergebnis steht. Hier wäre mehr ärztliche Zurückhaltung gefragt.
}

_ Die Gynäkomastie ist die häufigste Veränderung der männlichen Brustdrüse. Obwohl es auch hier zu Karzinomen kommen kann, ist die Häufigkeit um mehrere Größenordnungen geringer als im Falle der weiblichen Brust. Trotzdem betrachten viele Ärzte eine Bildgebung als essenziellen Bestandteil der Diagnostik.

Dass dies nicht gerechtfertigt ist, zeigt eine retrospektive Untersuchung an 557 Patienten, die in den letzten zehn Jahren in der radiologischen Abteilung des Amsterdamer Universitätsklinikums untersucht wurden. $74 \%$ wurden wegen einer Gynäkomastie oder Schwellung, 24\% wegen Schmerzen und 10\% wegen Knotenbildung überwiesen. Bei $75 \%$ der Patienten wurden eine Mammografie, bei $51 \%$ eine Ultraschalluntersuchung und bei $26 \%$ beide Verfahren eingesetzt. Nach der BI-RADS-Klassifikation des American College of Radiology wurde bei 519 Patienten ein Befund Klasse 1 oder 2 erhoben, also ein Normalbefund oder ein sicher benigner Befund. 38 Patienten wiesen BI-RADS 3 oder höher auf. Der Einsatz von Feinnadelaspirations-Zytologie oder Biopsie bei 160 Patienten ergab nur in fünf Fällen $(0,89 \%)$ einen malignen Befund. Bei diesen fünf Patienten war allerdings auch die klinische Untersuchung bereits verdächtig auf das Vorliegen eines Malignoms gewesen.

Dagegen wurde bei keinem einzigen Patienten zufällig ein asymptomatisches Malignom entdeckt. Die bildgebenden Verfahren hatten eine Sensitivität von $80 \%$ und eine Spezifität von $99 \%$. Der positive prädiktive Wert errechnete sich mit $44 \%$, der negative prädiktive Wert mit 90,8\%. Die Wahrscheinlichkeit eines Malignoms der männlichen Brustdrüse ist so gering, dass die Autoren vom Einsatz jeder Bildgebung bei der Gynäkomastie des Mannes abraten.

- Lapid O, Siebenga P, Zonderland HM. Overuse of imaging
the male breast - findings in 557 patients.

Breast J. 2015;21:219-23

\section{KOMMENTAR}

Gerade weil die männliche Brustdrüse einer klinischen Untersuchung so gut zugänglich ist, sollte man seinem klinischen Urteil voll vertrauen und nur bei solchen Patienten eine Bildgebung initiieren, die tatsächlich einen Tastbefund aufweisen, der über die bloße Vergrößerung der Brustdrüse hinausgeht. Das ist wieder ein Punkt auf der immer länger werdenden Liste der ärztlichen Kunst des Unterlassens.

Prof. Dr. med. H. S. FüeßI

\section{Lindsay-Nägel bei chronischer Niereninsuffizienz}

Eine 59-jährige Frau suchte wegen seit etwa drei Jahren bestehender Nagelveränderungen ärztlichen Rat. Aus der Vorgeschichte war bemerkenswert, dass die Frau sich vor 16 Jahren wegen einer polyzystischen Nierenerkrankung einer Nierentransplantation hatte unterziehen müssen.

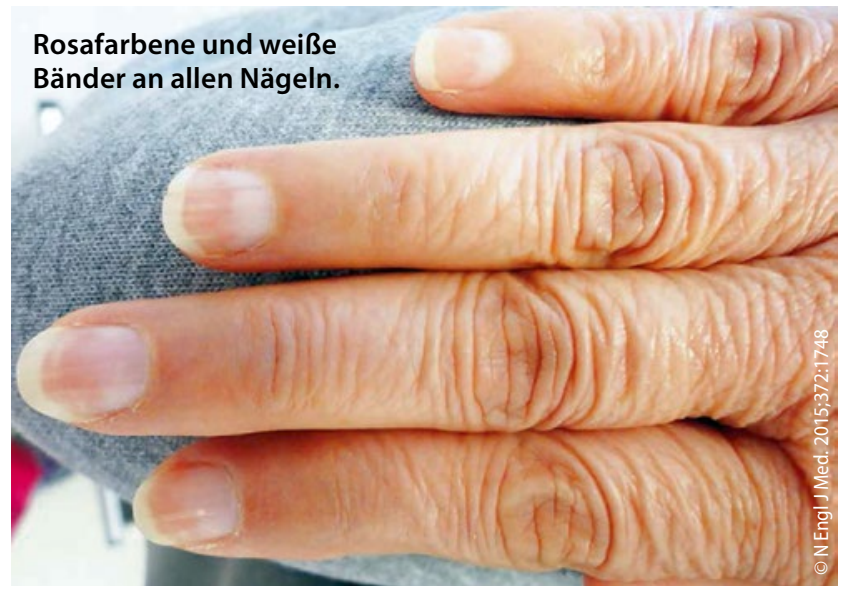

Deshalb stand sie auch unter einer Dauertherapie mit Prednison, Mykophenolat und Takrolimus. Sie wies das Stadium 4 einer chronischen Niereninsuffizienz auf, ihre Kreatininwerte lagen im vergangenen Jahr zwischen 1,7 und 2,2 mg/dl und ihr Harnstoffwerte zwischen 26 und 38 $\mathrm{mg} / \mathrm{dl}$. An sämtlichen Fingernägeln fand sich ein rosafarbenes, quer verlaufendes Band, das etwa 50\% des Nagelbetts einnahm. Mehr distal zum Nagelfalz hin zeigte sich ein weißes Band.

Lindsay-Nägel oder Halb-und-halb-Nägel wurden 1967 erstmals als rotes, rosafarbenes oder braunes Band, das 20-60\% des Nagelbetts einnimmt, bei Patienten mit chronischer Nierenerkrankungen beschrieben. Es gibt keine Korrelation zwischen dem Ausmaß der Veränderungen und dem Grad der Azotämie. Das proximal gelegene weiße Band ist durch die renale Anämie bedingt, das distal gelegene braune Band durch MelaninAblagerungen. Diese kommen wahrscheinlich durch erhöhte Konzentrationen von $\beta$-Melanozyten-stimulierendem Hormon zustande. Abgesehen von der Behandlung der zugrunde liegenden Nierenerkrankung gibt es keine spezifische Therapie.

Prof. Dr. med. H. S. FüeßI

- Yang CS, Robinson-Bostom L (catherine_yang@brown.edu). Lindsay's nails in chronic kidney disease. NEngl J Med. 2015;372:1748 